\title{
DEL NUDGE Y LA LIBERTAD DE ELEGIR ${ }^{1}$
}

\author{
About Nudge and Choice Freedom
}

\author{
JoNATHAN ECHEVERri ÁlVVAREZ², IsABEl LOPERA ArbELÁEZ ${ }^{3}$
}

https://doi.org/10.17533/udea.rp.v12n1a08

\begin{abstract}
“Cómo mantenerme libre? ¿Cómo preservar, a pesar de todas las amenazas y todos los peligros, en medio de la furia de los bandos en lucha, la insobornable claridad del espíritu, y cómo conservar ilesa la humanidad del corazón en medio de la bestialidad? ¿Cómo sustraerme a las exigencias que el Estado o la Iglesia o la política me quieren imponer contra mi voluntad? ¿Cómo defenderme para no ir en mis palabras y acciones más allá de donde mi yo más íntimo quiere llegar? ¿Cómo proteger esta parcela única y particular de mi yo, que en un rincón único me refleja el universo, contra la sumisión a la mesura reglamentada y decretada desde afuera?

Por amor a esta libertad, se observaba a sí mismo, se vigilaba, examinaba... en cada uno de sus actos y pensamientos. Ni el más sabio escapa a la tentación. Primero quiere conocerse; después, mostrarse como es".
\end{abstract}

Zweig (2008, 19-20)

\section{Resumen}

Este artículo de reflexión expone una perspectiva filosófica y empírica, sobre un debate contemporáneo, a propósito de la intervención de la toma de decisiones. Para esto nos ocuparemos de tres problemas básicos: el pa-

Recibido: 02-07-2020 / Aceptado: 21-08-2020

Para citar este artículo en APA: Echeverri, J. y Lopera, I. (2020). Del nudge y la libertad de elegir.

Revista de Psicología Universidad de Antioquia, 12(1), 157-178. doi: https://doi.org/10.17533/ udea.rp.v12n1a08 ternalismo libertario; del nudge y la libertad de elegir; y la potencial interacción entre diseño de contexto y formación de autonomía. En el primer problema discutiremos una posible actualización conceptual y empírica del ensayo 
Dos conceptos de libertad de Berlin (1988); el segundo apartado desarrolla la idea propuesta por Thaler y Sunstein (2009) del nudge como estrategia para direccionar la decisión: finalmente, proponemos una intervención de la decisión humana que se ocupe de los dos ti- pos de libertad según Berlin (1988), esto es, la libertad positiva y la libertad negativa.

Palabras clave: toma de decisiones y psicología, toma de decisiones éticas, autonomía moral, teoría de la decisión.

\section{Abstract}

This paper presents a philosophical and empirical perspective, on a contemporary debate, about the intervention in decision making. For this we will deal with three basic problems: libertarian paternalism; the nudge and the decision maker; and the potential interaction between context design and autonomy formation. In the first problem we will discuss a possible conceptual and empirical update of the essay Two concepts of freedom of Berlin (1988); In the second section, we develop the idea proposed by Thaler and Sunstein (2009) of nudge as a strategy for directing the decision. Finally, we propose an intervention of the human decision that deals with the two types of freedom according to Berlin (1988), that is, positive freedom and negative freedom.

Keywords: decision making and psychology; ethical decision making; moral autonomy.

\section{Introducción}

El advenimiento de la economía conductual en segunda mitad del siglo xx supuso un cambio conceptual de la racionalidad económica a la racionalidad limitada. De la creencia según la cual el agente decisor es racional, a la conjetura empírica de un agente razonable con importantes limitaciones psicológicas para tomar decisiones. En la primera creencia, la teoría económica supone que los agentes (decision-makers) son racionales (escogen siempre la mejor opción disponible a través de un análisis costo-beneficio), cuentan con habilidades de pensamiento que permiten estimar con precisión probabilidades, se rigen por intereses egoístas y, en el momento de realizar una elección económica, están desprovistos de cualquier emoción e impulso altruista que pueda enturbiar, aparentemente, el curso óptimo de los acontecimientos. Un agente racional es aquel que procura, sistemáticamente, maximizar el resultado óptimo de sus preferencias (Kahneman 2003; Pena 2005). Tal compendio de supuestos normativos se enmarca en un modelo ético utilitarista; además, 
permite una formalización matemática. En la teoría económica no ha sido posible, por ejemplo, formalizar tanto la incidencia de cualquier tipo de reacción visceral en la decisión como los cambios imprevistos de motivaciones y preferencias humanas, aunque existe una pretensión constante de incluir estos aspectos de la decisión —como la incidencia de la emoción y la intuición- en modelos matemáticos de mayor complejidad explicativa (Berridge y Aldridge 2008). En este sentido, es comprensible el interés por los supuestos descritos en el modelo del agente racional (Ariely 2008). Por otra parte, la noción de racionalidad limitada constituye el punto de partida que enfatiza en el mecanismo de la intuición para emitir juicios y elegir. Esta tesis ha desencadenado múltiples investigaciones que consolidan el campo de la economía conductual, en consonancia con el desarrollo de otros campos como la psicología moral, al tratar algunos de los siguientes temas: corrupción, conflicto de interés, cooperación, entre otros (Ariely, 2008 2011; Bowles, 2011; Thaler y Sunstein, 2009).

En el presente artículo, presentamos un diálogo filosófico y empírico con el propósito de explorar las posibilidades de intervención de la decisión humana. Proponemos una intervención que se ocupe de los dos tipos de libertad según Berlin (1988), esto es, la libertad positiva y la libertad negativa. Sin desconocer la investigación empírica en psicología, que en la segunda mitad del siglo xx muestra la presencia de error en la elección, ¿cómo formar la autonomía o libertad positiva en relación con los cambios en contexto que se logran con la intervención? En últimas, la injerencia en la decisión humana es posible en la medida que nos ocupemos de forma simultánea de la autonomía y la heteronomía.

\section{Paternalismo libertario}

La libertad negativa y la libertad positiva representan dos concepciones, como ideal filosófico normativo, que reaparecen con el enfoque de intervención propuesto por Thaler y Sunstein (2009) denominado paternalismo libertario. La libertad negativa implica heteronomía, no interferencia, ausencia de coacción; en cambio, la libertad positiva constituye autonomía, dirección 
autonormativa. Estos dos ideales, según la distinción propuesta por Berlin (1988), exhiben perspectivas que han tenido un desarrollo histórico simultáneo y opuesto. En un escenario resulta más importante no interferir en las decisiones de otras personas, y en el otro escenario es importante reconocer la capacidad que tienen los seres humanos para labrar su propio destino en función de sus preferencias. El paternalismo libertario desconfía del segundo escenario, pues supone, sobre la base de investigación empírica en economía conductual, que las personas habitualmente toman decisiones perjudiciales para sí mismas. De forma generalmente inconsciente las decisiones se toman de forma automática y son sensibles a cambios que acontecen en el entorno, en detrimento incluso del desarrollo humano, el bienestar y la calidad de vida (Ariely, 2008; Kahneman, 2003, 2012; World Bank Group, 2015). El paternalismo libertario confía en la posibilidad de direccionar la vida de las personas sin restringir ninguna de sus elecciones. Una intervención política grupal o de gran envergadura es paternalista en la medida que supone por antelación la dirección beneficiosa que deben tomar las decisiones de las personas, y es libertaria en tanto que no restringe aquellas opciones que pueden afectar de forma negativa la vida.

$\mathrm{Al}$ asumir una posición paternalista y libertaria, ¿cuál es el argumento que justifica promover una decisión en cierta dirección? ¿Cómo evitar la coerción política cuando se establece con anticipación la mejor opción posible a elegir? ¿Cuál es el punto de encuentro o desencuentro entre promover una decisión y permitir elegir cualquier alternativa? Berlin (1988) señala que precisamente la coacción se justifica señalando con antelación un fin; en sus palabras, la coacción política sugiere que "[...] es posible, y a veces justificable, coaccionar a los hombres en nombre de algún fin (v.g. la justicia o la salud pública) que ellos mismos perseguirían, si fueran más cultos, pero que no persiguen porque son ciegos, ignorantes o están corrompidos" (p. 9). De esta manera, la imposición u obligación emerge con su fuerza política porque alguien supone saber qué resulta ser mejor para los demás. En otras palabras, alguien sugiere e impone desde la coacción una ruta de elección, con un supuesto valor preferencial superior al de las demás opciones. En esta dirección se orientan las primeras objeciones (Gigerenzer, 2015a; Grüne-Yanoff y Hertwig, 2015; Grüne-Yanoff 
2016) en contra del paternalismo libertario propuesto por Thaler y Sunstein (2009). Esta idea de intervención, que se deriva de la economía conductual, incorpora de forma implícita una discusión filosófica moderna en torno a la libertad: una actualización empírica y conceptual del ensayo Dos conceptos de libertad de Berlin (1988). De acuerdo con Thaler y Sunstein (2009), y a propósito de la aparente coacción que promueve el enfoque, en primer lugar, no es posible evitar incidir en las decisiones de las personas en ninguna circunstancia. En variadas situaciones, y debido a nuestras formas complejas de organización social, las decisiones individuales y la disposición del contexto, en cualquiera de sus formas, afectan de forma directa o indirecta las decisiones de otras personas.

En esta perspectiva, todo contexto grupal u organizacional incide de forma consciente o inconsciente en las decisiones de sus miembros. A este contexto se le nombra arquitectura para la toma de decisiones (Sunstein, 2017; Thaler y Sunstein, 2009). Un contexto que promueve o censura las elecciones de los individuos que lo componen. No es posible concebir una elección desprovista de contexto (Sunstein, 2017). De forma inevitable, el ser humano enfrenta restricciones o posibilidades, presentes en la arquitectura, que interaccionan con su respectivo ejercicio de libertad. En segundo lugar, el paternalismo libertario, según los autores, no implica coacción en tanto no se restringe ninguna alternativa de respuesta. Y esto porque "los riesgos que entrañan los diseños ineptos e incluso corruptos se reducen manteniendo las opciones sin restricciones. La libertad de elección es la mejor salvaguardia contra una mala arquitectura de las decisiones" (Thaler y Sunstein, 2009, p. 26). La decisión se orienta a una dirección específica sin eliminar alguna posibilidad que exista en detrimento del bienestar. Ahora bien, en el contexto de la libertad negativa, Berlin (1988) resalta que:

La mera existencia de dos posibilidades no es, por tanto, suficiente para hacer que mi acción sea libre (aunque puede que sea voluntaria) en el sentido normal que tiene esta palabra. La amplitud de mi libertad parece depender de lo siguiente: a) de cuántas posibilidades tenga [...]; b) de qué facilidad o dificultad haya para realizar estas posibilidades; c) de qué importancia tengan éstas, comparadas unas con otras, en el plan que tenga de mi vida, dados mi carácter y circunstancias; d) de hasta qué punto estén abiertas o cerradas por los actos deliberados que ejecutan los 
hombres; e) de qué valor atribuyan a estas varias posibilidades, no sólo el que va a obrar, sino también el sentir general de la sociedad en que éste vive. Todas estas magnitudes tienen que «integrarse», y de este proceso hay que sacar una conclusión, que no es nunca necesariamente precisa ni indiscutible. Bien puede suceder que haya muchos tipos y grados inconmensurables de libertad y que éstos no se puedan determinar en una sola escala de magnitud. Más aún, en lo que se refiere a las sociedades nos enfrentamos con cuestiones (absurdas lógicamente) como esta: ¿aumentaría la situación x la libertad del señor a más que la que tienen entre sí los seńores B, C y D sumados todos juntos? (p. 8).

En síntesis, el oportuno ejercicio de esta libertad negativa depende del grado de complejidad de la situación que exija una decisión -la primera, segunda, tercera y cuarta descripción que expone Berlin (1988) en el pasaje citado- y del nivel de conocimiento que cada persona tenga de sus preferencias — quinto rasgo o descripción del autor-. Esta última descripción remite también a la autonomía.

En este texto, sugerimos la necesidad de articular una intervención orientada en ambos frentes. Esto es, diseñar entornos amigables para simplificar el arduo proceso de tomar una decisión en situaciones complejas (libertad negativa o heteronomía); propiciar espacios que permitan a las personas reflexionar y mejorar su autonomía, en tal caso consideramos la libertad positiva como una capacidad susceptible de formarse, una capacidad que permite imaginar y realizar una oportunidad aún cuando no esté presente en el contexto y al tiempo una disposición que se potencia en entornos complejos a través de la experiencia (libertad positiva o autonomía); y orientar intervenciones adaptables hacia la construcción de un canal de comunicación entre el diseño de contexto y la formación de autonomía; es decir, propiciar oportunidades y sugerirlas, a la vez que se confieren espacios para reflexionar y formar-se en capacidad para tomar decisiones (interacción entre heteronomía y autonomía).

La tercera forma de intervención es polémica en tanto sugiere una potencial interacción entre dos concepciones de libertad contrapuestas y dos nociones de la decisión humana divergentes. En el primer caso, la heteronomía, en la perspectiva contemporánea que imprime el paternalismo libertario, se asume que las personas habitualmente toman decisiones perjudiciales para sí mismas, por tal razón necesitan ayuda; en el segundo caso, la autonomía, 
se hace énfasis en la capacidad del ser humano de elegir sin requerir algún tipo de intervención externa. Resaltamos dos escuelas que hacen investigación en ambas direcciones de forma paralela, la iniciativa propuesta por autores como Ariely (2008, 2011), Kahneman, (2003, 2012), Sunstein (2017), Thaler (2016) y Thaler y Sunstein (2009), en dirección al cambio de contexto, que se materializa en agencias de investigación aplicada como The Behavioral Insights Team en Reino Unido o Ethical Systems en Nueva York, y, por otro lado, el programa de investigación propuesto por Gigerenzer (2015a, 2015b) The Center for Adaptative Rationality (ARC) del Max Planck Institute For Human Development en Berlín, enfocado en la formación de capacidad para tomar decisiones en incertidumbre, sin recurrir al cambio de contexto. En los siguientes dos apartados haremos una exposición mayor de esta posible interacción entre ambos enfoques.

\section{Del nudge y la libertad de elegir}

Nudge es un pequeño empujón, "es cualquier aspecto de la arquitectura de la toma de decisiones que modifica la conducta de las personas de una manera predecible sin prohibir ninguna opción ni cambiar de forma significativa sus incentivos económicos" (Thaler y Sunstein, 2009, p. 20). No representa un incentivo, exigencia o prohibición, es un cambio singular de contexto cuyo propósito es sugerir una decisión en una dirección específica (paternalismo). Este cambio de contexto no elimina o retringe opciones (libertarismo), presenta de forma preferible una alternativa sobre otra. Algunos ejemplos: modificar la disposición de alimentos en la comida para preferir las verduras y así obtener una alimentación saludable; asumir compromisos financieros o no financieros para cumplir un objetivo concreto como bajar de peso o realizar una tarea específica que se aplaza de forma indefinida, en caso de no cumplir con el objetivo, en un plazo determinado, es necesario pagar el compromiso financiero o no financiero que ha sido asumido; tener alguna aplicación en un dispositivo móvil que indique los potenciales beneficios de utilizar movilidad sostenible para desplazarse en la ciudad y reportar información en tiempo real al usuario en torno a variables como peso y contaminación evitable en el lugar 
que se habita; enviar mensajes cortos al dispositivo móvil o computador a pacientes, en situación delicada de salud, para que tomen sus medicamentos a tiempo y así cumplir con el regimen médico indicado, entre otros.

Estos nudges o cambios de contexto en principio son imaginados por un arquitecto de la decisión, es decir, una persona (o también un grupo de personas) que diseńa la arquitectura para la toma de decisiones en un contexto social específico, luego se realiza un seguimiento experimental al nudge sugerido por el arquitecto. Una vez se implementa el nudge es indispensable identificar de forma sistemática los cambios acontecidos en las decisiones de las personas. ¿Qué cambios ocurren ante la presencia de un nudge en un grupo de personas, en comparación con otro grupo con características muestrales idénticas que no experimenta el cambio de contexto? Esta inquietud lidera la investigación aplicada cuyo objeto es el diseño e implementación de nudges. Tal perspectiva supone que las decisiones humanas están inmersas en un entramado de efectos contextuales que las guían en una u otra dirección; la complejidad de estos efectos contextuales (por diversos y numerosos) y la incapacidad del ser humano, por su "racionalidad limitada" (Simon, 1978, 2000), para evaluar con detenimiento las opciones y elegir de forma razonable aquella que considere mejor, constituyen dos razones que justifican la intromisión de una postura paternalista. De acuerdo con Ariely (2008):

[...] la capacidad de reconocer lo que nos separa del ideal constituye una parte importante de la empresa de tratar de conocernos realmente a nosotros mismos, y una parte, además, que promete numerosos beneficios prácticos. Comprender la irracionalidad es importante para nuestras acciones y decisiones cotidianas, $y$ también para entender la forma en que diseñamos nuestro entorno y las opciones que éste nos presenta (p. 17).

La postura de Ariely (2008) sugiere la necesidad de conocer nuestra irracionalidad, nuestra tendencia permanente al error en la elección, con el propósito de tener apoyo, esto es, herramientas que mejoren el proceso de toma de decisiones. De la misma forma como nuestras limitaciones físicas motivan el diseño y creación de herramientas para convivir con estas restricciones (el automóvil en el desaplazamiento de grandes distancias o el celular para comunicarnos en ausencia de cercanía física), nuestras limitaciones de pensamiento, 
vulnerables a los efectos contextuales, deben motivar la existencia de recursos para convivir con estas restricciones de elección.

Desde la segunda mitad de siglo xx, la psicología ha manifestado un creciente interés por ofrecer modelos explicativos que se desprendan de una descripción rigurosa en torno al proceso psicobiológico que subyace a una decisión, sin necesidad de asumir, de entrada, una postura normativa desde la teoría económica. Semejante interés permitió el surgimiento de la economía conductual (Kahneman, 2003, 2012; Smith, 2005). Los supuestos presentes en la figura del homo economicus se convirtieron en las hipótesis a contrastar experimentalmente. La noción de homo economicus o agente racional, se ha justificado en la historia del pensamiento económico por constituir una imagen del hombre en la economía descentralizada del capitalismo, y ofrecer atajos metodológicos relevantes para la teoría. Es decir, con el advenimiento de las relaciones económicas capitalistas propias del Reino Unido en el siglo XviII, el trabajo y el espacio laboral emergen como "esferas temporales que se diferenciaban de la familia, la religión, la política y las costumbres. Se decía que una psicología en particular — resumida por los economistas clásicos como el "hombre económico" calculador, interesado en sí mismo, y amoral-, gobernaba nuestro comportamiento en este espacio económico" (Bowles, 2010, p. 5). De igual manera, esta concepción del hombre se adecuó fácilmente al razonamiento matemático de los modelos económicos, en tanto que permitía simplificar las dinámicas de la elección a un comportamiento interesado y egoísta, libre de cualquier otra circunstancia que pudiese hacer impredecible una decisión económica.

A raíz de este interés, y de los hallazgos experimentales que han sido obtenidos, Kahneman (2012) concluye que "los seres humanos no están bien descritos en el modelo del agente racional” (p. 535) y precisa esta idea con lo siguiente observación:

Los científicos sociales de la década de 1970 aceptaban generalmente dos ideas acerca de la naturaleza humana. La primera era que la gente es generalmente racional, y su pensamiento normalmente sano. Y la segunda, que emociones como el miedo, el afecto o el odio explican la mayoría de las situaciones en las que la gente se aleja de la racionalidad. Nuestro artículo desafiaba a estas dos suposiciones [...] Documenta- 
mos de manera sistemática errores en el pensamiento de la gente normal y buscamos el origen de dichos errores en el diseño de la maquinaria de la cognición más que en la alteración del pensamiento por la emoción (Kahneman, 2012, p. 20)ㄹ.

El interés creciente por esta documentación sistemática sobre posibles errores del pensamiento en la elección y la inconformidad, que ha generado el modelo del agente racional en interior de la comunidad académica, da lugar al nacimiento de un nuevo proyecto científico que pone en relación la psicología con la economía. El carácter polémico de la economía conductual reside en la supuesta demostración de que los seres humanos, a diferencia de la habitual idea de racionalidad en la teoría económica ortodoxa, tienden a ser irracionales; es decir, las decisiones no suelen ser óptimas en función de los recursos e información disponible y, por ende, no son ejecutadas de acuerdo a la posible persecución de un beneficio directo aunque así lo parezca. Las decisiones en esta perspectiva suelen considerarse como previsiblemente irracionales (Ariely, 2008, 2011). En otras palabras, errores evidentes en las decisiones cotidianas no sólo hacen pensar en la necesidad de adjudicar el rótulo de irracionalidad, también la manifestación sistemática de estos hacen posible su respectiva previsión.

¿Es posible atribuir a la economía conductual la demostración aparente de lo estrictamente irracional? ¿Los modelos económicos convencionales resisten la inclusión de una psicología del agente irracional? Si bien las investigaciones de Kahneman y Tversky, iniciadas en la década de 1970, utilizaron como hipótesis nula el modelo del agente racional, los resultados obtenidos no fueron categorizados bajo la etiqueta de lo irracional; a pesar de los errores sistemáticos en razonamiento claramente demostrados. De acuerdo con Kahneman (2003:

3 El artículo que señala Kahneman fue publicado en colaboración con Amos Tverstky en Science, 185, 1974, con el título "El juicio bajo incertidumbre: heurísticas y sesgos". Éste y otro texto publicado entre ambos autores (cf. "Elecciones, valores y marcos", en American Psychologist, 34, 1984) dieron lugar a la concesión del Premio Nobel en Economía en el año 2002. Se les atribuye el surgimiento de un nuevo ámbito de estudio, esto es, la economía conductual (Behavioral Economics) que relaciona la teoría económica con observaciones y experimentos psicológicos sobre la decisión en condiciones de incertidumbre (Kahneman, 2003, 2012). 
La característica fundamental de los agentes no es la de que razonan mal sino la de que actúan a menudo intuitivamente. Y la conducta de estos agentes no está guiada por lo que son capaces de calcular, sino por lo que por casualidad ven en un momento dado (p. 215).

Esta consideración suele omitirse en conferencias y publicaciones que intentan popularizar los resultados obtenidos en este campo, además de las usuales interpretaciones que han realizado algunos psicólogos y economistas (Ariely, 2008, 2011; Grupo Banco Mundial, 2015; Smith, 2005). La contribución de la psicología a la economía consiste en integrar un mapa de racionalidad limitada a los estudios de las preferencias económicas. Un mapa que describe la arquitectura de la cognición que involucra el funcionamiento de la percepción, la intuición y el razonamiento (Stanovich y West, 2000), sin excluir la importancia de las emociones.

Las ideas que han guiado la agenda programática de investigación en economía conductual pueden resumirse en las siguientes dos tesis: la primera sostiene que, generalmente, los juicios y las elecciones se realizan de manera intuitiva; la segunda, que los principios que gobiernan la intuición son semejantes a los de la percepción, por consiguiente, el acervo teórico disponible en psicología sobre el fenómeno de la percepción sirve para entender la naturaleza de los juicios intuitivos (Kahneman, 2003). Ante una posible elección puede apelarse a un proceso lento, controlado y con esfuerzo propio del razonamiento; o a un proceso rápido, automático y sin esfuerzo característico de la percepción e intuición.

La intuición dispone de características de la percepción, pero no se limita al procesamiento del estímulo, funciona en relación con percepciones y conceptos acumulados que pueden ser evocados, ubicándose entre "las operaciones automáticas de la percepción y las operaciones deliberadas del razonamiento" (Kahneman, 2003, p. 186). Estos resultados sobre la intuición no limitan ampliamente, como pudiese parecer, los trabajos conceptuales y aplicados en relación a la elección económica. Constituye un punto de referencia sobre el cual las líneas de investigación se han desarrollado utilizando evidencia proveniente de las ciencias cognitivas, de la psicología evolutiva y de la psicología social en un plano experimental. Las observaciones de 
Kahneman (2003) apuntan a la importancia de incorporar un agente intuitivo o razonable a los modelos económicos donde la separación tradicional entre las creencias - concepto amplio que hace alusión a diversas características como la emoción, el optimismo y expectativa-y las preferencias, en los análisis económicos, es inadmisible.

En síntesis, la economía conductual (Behavioral Economics) surge de la investigación en psicología básica proponiendo un modelo de elección alterno al disponible en la teoría económica. Esta agenda de investigación incorpora a los modelos económicos fenómenos como el altruismo; la inconsistencia temporal de las preferencias; la coherencia arbitraria en individuos tomadores de precios; disonancia cognitiva e ilusión monetaria; entre otros (Kahneman, 2003, 2012; Smith, 2005)4. Estos aspectos configuran el modelo del agente razonable o agente con racionalidad limitada, y que por lo tanto requiere la presencia de algún nudge, en contraposición al "agente racional”, la diferencia entre ambas denominaciones al parecer es sutil pero describe unas distinciones conceptuales importantes, que ya han sido descritas, en la forma de explicar la elección racional.

Los rasgos característicos de esta racionalidad limitada son descritos de forma diversa. Vislumbrar tales rasgos es de vital importancia para el diseño e implementación de algún nudge. Por ejemplo, el Informe sobre Desarrollo Humano Mundial publicado por el Grupo Banco Mundial (2015) postula la exis-

4 En forma paralela la disciplina económica impulsó la economía experimental en la segunda mitad del siglo xx. A diferencia de la economía conductual el punto de partida no fue el modelo clásico del agente racional, puesto que su única pretensión en un inicio fue el uso de experimentos en el análisis económico convencional como herramienta metodológica de apoyo. No obstante, el oficio de esta disciplina en el diseńo óptimo de experimentos ha permitido encontrar resultados en el laboratorio cada vez más interesantes para la teoría económica y el estudio del comportamiento humano en un gran número de elecciones. La referencia más relevante en este reciente campo de la economía es Vernon L. Smith, a quien se otorgó el Premio Nobel del año 2002. El modelo experimental que subyace a sus conocidos estudios consiste en la interrelación de tres elementos: la institución (reglas de intercambio en el mercado, reglas de intercambio de información, etc.), el entorno (conocimiento, valores, y dotación de recursos del agente, entre otros) y el comportamiento a examinar. En este caso el investigador controla y manipula la institución y el entorno para analizar el comportamiento del agente, resultado de los múltiples estados en las variables independientes descritas (Moreno, 2002). Desde un principio, Smith (2005) con su inicial publicación experimental titulada An Experimental Study of Competitive Market Behavior (1962), el modelo del agente racional no constituye una referencia teórica problemática. El objetivo principal de la propuesta metodológica ha consistido en contrastar en el laboratorio las hipótesis de la teoría económica neoclásica. 
tencia de tres principios en las decisiones humanas: el pensamiento automático, el pensamiento social y el pensamiento basado en modelos mentales. El pesamiento automático refiere a la adopción de juicios y decisiones de forma automática sin necesidad de deliberar, este hace referencia al proceso rápido característico de la intuición. El pensamiento social implica que los juicios y las decisiones de una persona son fácilmente influenciados por juicios y decisiones de otras. Y el pensamiento basado en modelos mentales implica tener una perspectiva en común, con otros seres humanos, de sí mismo y el entorno. Estos tres rasgos del pensamiento explican la dependencia "irracional" o más bien intuitiva a cambios contextuales. Por su parte Thaler y Sunstein (2009), mencionan la racionalidad limitada, la falta de autocontrol y la dependencia a la influencia social, como aspectos del comportamiento humano que explican decisiones económicas que, en retrospectiva, han motivado incluso la existencia de crisis económicas y políticas (ver por ejemplo el caso de la crisis inmobiliaria y financiera del año 2008 presentada por ambos autores en su libro). Ante el diseño de algún nudge y su posible justificación normativa, el Grupo Banco Mundial (2015) sugiere plantearse la siguiente pregunta, desde la cual expone tres razones a manera de respuesta "ipor qué los Gobiernos deberían ocuparse de configurar las elecciones individuales?” (pp. 21-22):

\footnotetext{
[...] En primer lugar, al configurar las elecciones se puede ayudar a los individuos a lograr sus propios objetivos. [...] En segundo lugar, las preferencias y las metas inmediatas de los individuos no siempre se corresponden con sus propios intereses. [ ...] En tercer lugar, las prácticas y los modelos mentales que se refuerzan socialmente pueden bloquear elecciones que generan mayor capacidad de acción y promueven el bienestar y, por ende, pueden impedir que los individuos conciban siquiera ciertos cursos de acción, como cuando la discriminación lleva a las personas a adoptar, comprensiblemente, aspiraciones poco ambiciosas. Los Gobiernos deberían tomar medidas cuando la interacción inadecuada, la configuración de la situación y las prácticas sociales socavan la capacidad de acción del individuo y generan o perpetúan la pobreza.
}

La concepción del nudge y la necesidad de su respectiva implementación se desprende de una forma singular de concebir al agente que toma decisiones. La modificación o cambio de contexto sugiere al parecer un agente confinado al error. ¿ Es posible concebir otra alternativa que no requiera la intervención 
de algún gobierno? ¿Una alternativa de intervención que permita a los individuos desplegar su respectiva capacidad de decisión, al confiar en sus habilidades para mitigar el error y no depender, por lo tanto, de un continuo cambio de contexto? En el siguiente apartado, a raíz de una objeción conceptual y empírica de Gigerenzer (2015a, 2015b) al paternalismo libertario y al nudge por su dependencia exclusiva al cambio de contexto y su aparente concepción engañosa del error en la elección, presentamos una potencial interacción entre el diseño de contexto y formación de autonomía, en otras palabras, una intervención de la decisión humana que se ocupe de los dos tipos de libertad según Berlin (1988), esto es, de la libertad positiva y de la libertad negativa.

\section{Diseño de contexto y formación de autonomía}

La posibilidad de intervenir la decisión humana se debate, en la actualidad, entre el diseño de contexto y la formación de autonomía. Ambas alternativas son al parecer incompatibles. La objeción al paternalismo libertario, desde aquellos autores que propugnan la formación de autonomía, hace énfasis en las supuestas tres condiciones requeridas para que el arquitecto de la toma de decisiones realice su trabajo. Esto es, que en el arquitecto haya ausencia de sesgo y prejuicio, que comprenda la evidencia científica asociada con la elección y sus efectos y que no tenga conflicto de intereses en el momento de concebir alguna intervención (Barton y Grüne-Yanoff, 2015; Gigerenzer, 2015a; Grüne-Yanoff y Hertwig, 2015; Grüne-Yanoff, 2016). Al respecto, Gigerenzer (2015a, 2015b) insiste en resaltar estudios que demuestran el no cumpliento de estas condiciones y lo complejo que resulta para una sociedad garantizarlas. ¿Cómo garantizar, por ejemplo, la ausencia de conflicto de intereses cuando la implementación de algún nudge puede estar al servicio de cualquier beneficio económico o político encubierto? De ahí, por ejemplo, el uso de esta estrategia en campañas publicitarias de mercadeo y promoción política. Por tal razón, el autor propone diseñar programas de educación para el riesgo donde los ciudadanos puedan identificar la sutileza de estos cambios contextuales $y$, en consecuencia, tomen decisiones bien informadas. A esto se denomina alfabetización para el riesgo, contrario a la aversión al riesgo descrita por Kahneman 
$(2003,2012)$ en su teoría prospectiva de la utilidad. Desde esta perspectiva, la aversión al riesgo que se transforma continuamente ante cambios sutiles de preferencias, emociones e intuiciones, respecto a ganancias y pérdidas en un rango temporal, se modifica en el agente por una concepción del riesgo razonable que le permite, a través de una intuición educada o el uso consciente de heurísticos aprendidos, cuándo elegir una u otra opción en incertidumbre. Gigerenzer (2015a) señala que el paternalismo libertario desconfia de esta capacidad que tienen los seres humanos, a través de un proceso continuo de formación, de asumir riesgos de forma razonable con independencia de las posibles argucias y artimañas presentes en el contexto. En sus palabras:

\begin{abstract}
Como argumentaré con cierto detalle, el cuadro sombrío de la naturaleza humana pintado por los economistas conductuales y los paternalistas libertarios no está justificado por la investigación psicológica. Más bien, esto es en gran parte el producto de normas lógicas estrechas de racionalidad y de información selectiva de la literatura psicológica. Lo más importante para la política pública, al comparar las ilusiones cognitivas con las ilusiones visuales, los paternalistas libertarios sugieren engañosamente que los intentos de liberar a las personas de sus sesgos a través de la educación están en gran medida condenados al fracaso. Sin embargo, como mostraré, hay evidencia experimental de que incluso los niños pueden aprender a lidiar con el riesgo y la incertidumbre, si se les enseña cómo hacerlo. Llegaré a la conclusión de que los gobiernos democráticos deben invertir menos en empujar y más en educar a las personas para que se conviertan en expertos en el riesgo ${ }^{5}$ (Gigerenzer, 2015a, p. 364).
\end{abstract}

Desde el diseño de contexto, los autores responden a esta objeción señalando la importancia de proveer a las personas transparencia en la información en cualquier intervención, por ejemplo, "al poner de relieve los efectos del diseño de un plan sobre las decisiones que se toman, esperamos animar a los diseñadores a que se informen mejor" (Thaler y Sunstein, 2009, p. 260); evitar la

\footnotetext{
Traducción propia. Versión original: "As I will argue in some detail, the dismal picture of human nature painted by behavioral economists and libertarian paternalists is not justified by psychological research. Rather, it is largely the product of narrow logical norms of rationality and selective reporting of the psychological literature. Most important for public policy, by comparing cognitive illusions with visual illusions, libertarian paternalists misleadingly suggest that attempts to liberate people from their biases through education are largely doomed to fail. However, as I will show, there is experimental evidence that even children can learn to deal with risk and uncertainty - if they are taught how. I will conclude that democratic governments should invest less in nudging and more in educating people to become risk savvy" (Gigerenzer, 2015a, p. 364).
} 
asimetría de poder entre el arquitecto y el decisor (Mills 2015; Nagatsu 2015; Sunstein, 2015); y, concebir formas compatibles de incidir en la decisión, entre la heteronomía y el ejercicio de autonomía (Mill, 2015). En esta tercera vía, proponemos una intervención de la decisión humana que se ocupe de los dos tipos de libertad según Berlin (1988), esto es, la libertad positiva y la libertad negativa. El diseńo de contexto no es incompatible con la formación de autonomía, antes bien, puede promoverla. Esta última tampoco es consecuencia directa de una posible ausencia de restricción o no interferencia. Proporcionar feedback de elección aun en situaciones complejas y ambiguas y comprender las correspondencias entre elección y bienestar, representan posibilidades que permiten integrar dos escuelas de investigación que han tenido un desarrollo opuesto. La escuela orientada a la modificación de contexto y la escuela que hace énfasis en la alfabetización del riesgo como ejercicio de autonomía. Esta perspectiva requiere de una comprensión amplia de la decisión humana que, de un lado, reconoce que no existe una elección desprovista de contexto, o un supuesto decisor que cuenta con un criterio de valoración absoluta como resalta Ariely $(2008,2011)$, y, por tal razón, es tan susceptible de cambios ante la modificación contextual; y, de otro lado, explora las posibilidades de formación que los seres humanos tienen para educar su respectiva libertad positiva y su capacidad para tomar decisiones con autonomía, interpretando como punto de partida las sutilezas propias del contexto. ¿Cómo articular ambas dimensiones de la decisión en la intervención? Consideremos el siguiente punto de partida, en el escenario de la heteronomía:

Muchos economistas del desarrollo y especialistas en la materia creen que los elementos "irracionales" de la adopción de decisiones por el ser humano son inescrutables o que se cancelan mutuamente cuando interactúa un gran número de personas, como sucede en los mercados. Sin embargo, ahora sabemos que esto no es así. Las investigaciones de los últimos tiempos nos han permitido conocer mejor las influencias psicológicas, sociales y culturales a que están sujetas la toma de decisiones y la conducta de los seres humanos, y han demostrado que dichas influencias tienen un impacto significativo en los resultados en términos de desarrollo. Las investigaciones también revelan que es posible aprovechar estas influencias para alcanzar los objetivos de desarrollo. [...] Las investigaciones revelan que las pequeñas diferencias de contexto, conveniencia e importancia tienen grandes efectos en las opciones cruciales, como enviar a un niño a la escuela, prevenir una 
enfermedad o ahorrar para iniciar una actividad comercial. Esto signifca que los especialistas en el desarrollo deben centrarse no solo en determinar qué intervenciones son necesarias sino también en cómo se ponen en práctica. Ello, a su vez, exige que los organismos de ejecución dediquen más tiempo y recursos a la experimentación, el aprendizaje y la adaptación durante el ciclo de las intervenciones. (Grupo Banco Mundial, 2015, p. 5).

Las intervenciones necesarias, en este caso, a propósito del diseño e implementación de una política pública para el desarrollo humano, requieren un conocimiento previo de cómo los agentes deciden en sus elecciones habitualmente automáticas, orientadas por la influencia social y los estereotipos o modelos mentales. Esto aplica para diversos dominios de la experiencia humana como el cambio climático, elección política, pobreza, corrupción, cooperación y conflicto de interés, finanzas domésticas, entre otros. Los especialistas en este informe sugieren que, en primera instancia, los profesionales en desarrollo, responsables del diseño e implementación de una intervención, necesitan concebir estrategias para mitigar los efectos negativos de sus propias decisiones, luego, requieren iniciar un ciclo de intervención con las siguientes etapas: (1) Definir y diagnosticar; (2) Diseñar; (3) Implementar y evaluar; (4) Adaptar; (5) Redefinir y volver a diagnosticar.

El ciclo de intervención adquiere tal dinámica en tanto la evidencia experimental y el seguimiento sistemático de los efectos del nudge en las decisiones de las personas así lo requieran (Grüne-Yanoff, 2016). De esta forma, los diseños y las intervenciones son adaptables a cambios propios de decisiones individuales y colectivas. Las estrategias previas, al diseñar una intervención y diagnósticar un problema, para mitigar efectos negativos, indica que la persona (o grupos de personas) que concibe algún tipo de injerencia en las decisiones de otros no está exenta de cometer errores idénticos o similares a los errores que pretende corregir con el cambio de contexto, desde el diseño y el diagnóstico hasta la implementación y la evaluación del nudge. Desde la primera fase, la formación de autonomía es un escenario a considerar en la concepción del nudge e incluso en una modificación de contexto que favorezca el ejercicio de esta libertad positiva. Por ejemplo, ¿qué estrategias utilizar para mitigar el error y el conflicto de interés en el momento de diseñar un nudge? 
¿Y cómo disponer el contexto de tal forma que las personas interroguen sus procesos decisorios, inicien un proceso de formación de capacidad para decidir y asuman en consecuencia riesgos razonables? Esta es la dirección que posiblemente permita una interacción fructífera entre heteronomía y autonomía como dos perspectivas antiquísimas de la libertad, a veces contrapuestas. En el amplio espectro o ciclo de intervención "la inacción de los Gobiernos no necesariamente deja espacio a la libertad individual; por el contrario, puede representar una muestra de indiferencia frente a la pérdida de libertad" (Grupo Banco Mundial, 2015, p. 22).

\section{Conclusión}

La investigación contemporánea, en la conjución de disciplinas como la filosofía de la ciencia, la economía conductual y la psicología moral, concibe la decisión humana en incertidumbre como un centro de gravedad de interés disciplinario, es decir, que la toma de decisiones, objeto de indagación, permite el diálogo entre diversos enfoques que se ocupan de investigar diferentes dimensiones o expresiones de la misma (Díez, Guillén y Rodríguez, 2019; Echeverri, Lopera y Goenaga, 2016; Lopera, Echeverri y Goenaga 2019). Lograr explicar y comprender la decisión, en sus diversas manifestaciones culturales, y avisorar las posiblidades de intervención, es un campo de trabajo prometedor cuyo interés es potenciar el desarrollo humano (Álvarez, 2012; Cam, 2013; Lopera y Echeverri, 2018, 2019; Narvaez, 2014, 2015; Saldarriaga, 2010); el bienestar de las personas y las naciones (Sen, 1996; Tiberius, 1996; Vásquez, 2009a, 2009b); o la formación de los procesos decisorios individuales y colectivos que subyacen a una elección (Gigerenzer, 2015b; Mills, 2015; Naishtat, 1994); entre otros dominios de intervención (Echeverri y Gallego, 2019; Echeverri, Gallego y Silva 2019; Grupo Banco Mundial, 2015; Sunstein, 2017; Thaler y Sunstein, 2009). La distinción clásica de Berlin (1988) entre dos concepciones de libertad no sólo posibilita identificar dos ideas contrapuestas, en los discursos libertarios, de forma aparente; tal distinción también constituye un heurístico conceptual que en la actualidad ha permeado el desencuentro entre el diseño de contexto y la formación de autonomía para la toma de decisiones. 
Indagar sobre la posible interacción entre ambas perspectivas de intervención suscita múltiples inquietudes complejas y prometedoras. Basta señalar, por ejemplo, la posible articulación armónica entre presencia o ausencia de conflicto de interés, en el diseño de algún nudge, y la formación de autonomía para interrogar procesos decisorios y asumir riesgos razonables. Ocuparse de pensar e intervenir la libertad humana representa una inquietud que tiene un impacto en el desarrollo humano de las personas. Tal consideración propicia recordar los matices de las preguntas realizadas por Zweig (2008), a propósito del epígrafe utilizado en este texto.

\section{Referencias}

Ariely, D. (2008). Las trampas del deseo. Cómo controlar los impulsos irracionales que nos llevan al error. Barcelona: Editorial Ariel, S.A.

Ariely, D. (2011). Las ventajas del deseo: cómo sacar partido de la irracionalidad en nuestras relaciones personales y laborales. Barcelona: Editorial Ariel, S.A.

Álvarez, J. (2012). Amartya Sen. La concepción vigorosa de la libertad. Claves de Razón Práctica, 219, 76-82.

Barton, A. y Grüne-Yanoff, T. (2015). From Libertarian Paternalism to Nudging and Beyond. Review of Philosophy and Psychology, 6(3), 341-359. doi: https://doi. org/10.1007/s13164-015-0268-x.

Berlin, I. (1988). Cuatro ensayos sobre la libertad. Madrid: Alianza Editorial.

Berridge, K., y Aldridge, J. (2008). Decision Utility, The Brain, and Pursuit of Hedonic Goals. Social Cognition, 26(5), 621-646. doi: 10.1521/soco.2008.26.5.621.

Bowles, S. (2011). Microeconomía: comportamiento, instituciones, y evolución. Bogotá: Universidad de los Andes. Edición virtual.

Camps, V. (2013). Breve historia de la ética. Barcelona: RBA Libros S.A.

Díez, D., Guillén, M. y Rodríguez, M. (2019). Toma de decisiones éticas en organizaciones: una revisión de la literatura. Información Tecnológica, 30(3), 25-37.

Echeverri, J., Lopera, J., Goenaga, J. (2016). El cálculo racional para elegir o la deliberación con sabiduría. Revista Colombiana de Filosofía de la Ciencia, 16 (33): 113-137. 
Echeverri, J., Gallego, L. y Silva, S. (2019). Ciencias del comportamiento y gobernanza colaborativa: experiencias de cultura ciudadana en Medellín, Colombia. European Public \& Social Innovation Review (España), 4(1). 53-63.

Echeverri, J., Gallego, L. (Eds.) (2019). Cultura Ciudadana. Reflexiones y experiencias de ciudad. Medellín: Alcaldía de Medellín. Editorial Universidad Eafit.

Gigerenzer, G. (2015a). On the Supposed Evidence for Libertarian Paternalism. Review of Philosophy and Psychology, 6(3), 361-383. doi: https://doi.org/10.1007/ s13164-015-0248-1.

Gigerenzer, G. (2015b). Risk savvy. How to make good decisions. New York: Penguin Group.

Grüne-Yanoff, T. y Hertwig, R. (2015). Nudge versus boost: How coherent are policy and theory? Minds and Machines, 26(1-2), 149-183. doi: https://doi. org/10.1007/s11023-015-9367-9.

Grüne-Yanoff, T. (2016). Why behavioural policy needs mechanistic evidence. Economics and Philosophy, 32(3), 463-483. doi: https://doi.org/10.1017/ S0266267115000425.

Grupo Banco Mundial (2015). Informe sobre el Desarrollo Mundial: Mente, Sociedady Conducta. Washington, DC.

Kahneman, D. (2003). Mapas de racionalidad limitada. Psicología para una economía conductual. Revista Asturiana de Economía, 28, 181-225.

Kahneman, D. (2012). Pensar rápido, pensar despacio. Barcelona: Random House Mondadori, S.A.

Lopera, I. y Echeverri, J. (2018). Libertad y desarrollo humano en las organizaciones. Interdisciplinaria. Revista de Psicología y Afines (Argentina), 35(2), 395-408.

Lopera, I. y Echeverri, J. (2019). Decisiones, Bienestar y Desarrollo Humano en departamentos de bienestar estudiantil. Discusión de hallazgos. Estudos de Psicologia (Brasil), 24(3), 247-257. doi: https://10.22491/1678-4669.20190026.

Lopera, J., Echeverri, J. y Goenaga, J. (2019). De la sabiduría práctica y la decisión en incertidumbre. Universidad de Antioquia. Bogotá: Editorial Aula de Humanidades.

Mills, C. (2015). The Heteronomy of Choice Architecture. Review of Philosophy and Psychology, 6(3), 495-509. doi: https://doi.org/10.1007/s13164-015-0242-7.

Moreno, P. (2002). Premio Nobel de Economía de 2002: Vernon Smith y Daniel Kahneman. Boletín Económico de ICE, 2749, 25-36. 
Naishtat, F. (1994). Racionalidad, determinismo y tiempo en la decisión humana. En: Bleichmar, S. (Compiladora), Temporalidad, determinación, azar. Lo reversible y lo irreversible (pp. 149-182). Buenos Aires: Paidós.

Nagatsu, M. (2015). Social Nudges: Their Mechanisms and Justification. Review of Philosophy and Psychology, 6(3), 481-494. doi: https://10.1007/s13164-0150245-4.

Narvaez, D. (2014). Natural Morality, Moral Natures and Human Flourishing. In Musschenga, B. y van Harskamp, A. (Eds.). Why be moral? On the capacities and conditions for being moral. [Springer Library of Ethics and Applied Philosophy] Heidelberg, Germany: Springer.

Narvaez, D. (2015). Understanding flourishing: Evolutionary baselines and morality. Journal of Moral Education, 44(3), 253-262. doi: htt ps://10.1080/03057240.2015.1054619.

Pena, J. (2005). El problema de la racionalidad en economía o las inconsistencias del Homo oeconomicus. Estudios Filosóficos, 54(155), 33-57.

Rivera, J. (2007). La borrosa distinción riesgo-incertidumbre. Tecnológicas, 19, 13-46. doi: https://doi.org/10.22430/22565337.503

Saldarriaga, A. (2010). El sujeto activo: Antropología política en Amartya Sen. Eidos: Revista de Filosofía de la Universidad del Norte, 13, 54-75.

Sen, A. (1996). Capacidady bienestar. En: Nussbaum, M. y Sen, A. (Eds.). Calidad de vida (pp. 54-83). México: Fondo de Cultura Económica.

Simon, H. (1978). Rational decision-making in bussiness organizations. Nobel Memorial Lecture, 333-371.

Simon, H. (2000). Bounded rationality in social science: today and tomorroy. Mind and society, 1, 25-39. doi: https://doi.org/10.1007/BF02512227.

Sunstein, C. (2015). Nudges, Agency, Navigability, and Abstraction: A Reply to Critics. Review of Philosophy and Psychology, 6(3), 511-529. doi: https://10.1007/ s13164-015-0266-z.

Sunstein, C. (2017). Paternalismo libertario. Barcelona: Herder Editorial, S.L.

Smith, V. (2005). Racionalidad constructivista y ecológica en economía. Revista Asturiana de Economía, 32 197-273.

Stanovich, K. y West, R. (2000). Individual Differences in Reasoning: Implications for the Rationality Debate? Behavioral and Brain Sciences, 23, 645-726. doi: https://10.1017/S0140525X00003435. 
Tiberius, V. (2006). Well-Being: Psychological Research for Philosophers. Philosophy Compass, 1(5), 493-505. doi: https://doi.org/10.1111/j.17479991.2006.00038.x.

Thaler, R. (2016). Misbehaving. The Making of Behavioural Economics. UK: Penguin Random House.

Thaler, R. y Sunstein, C. (2009). Un pequeño enpujón (Nudge). El impulso que necesitas para tomar las mejores decisiones en salud, dinero y felicidad. Madrid: Santillana Ediciones Generales, S.L.

Vásquez, C. (2009a). La ciencia del bienestar psicológico. En: Vásquez, C. y Hervás, G. (Eds.). La ciencia del bienestar (pp. 13-46). Madrid: Alianza Editorial, S.A.

Vásquez, C. (2009b). El bienestar de las naciones. En: Vásquez, C. y Hervás, G. (Eds.). La ciencia del bienestar (pp. 103-142). Madrid: Alianza Editorial, S.A.

World Bank Group (2015). World development report: Mind, society and behavior. Washington, D.C.

Zweig, S. (2008). Montaigne. Barcelona: Acantilado. 